\title{
Air pollution, educational achievements, and human capital formation
}

\section{Exposure to elevated levels of air pollution adversely affects educational outcomes}

Keywords: air pollution, test scores, human capital, productivity, regulation

\section{ELEVATOR PITCH}

The link between air pollution and human health is welldocumented in the epidemiology and economic literature. Recently, an increasing body of research has shown that air pollution-even in relatively low doses-also affects educational outcomes across several distinct age groups and varying lengths of exposure. This implies that a narrow focus on traditional health outcomes, such as morbidity and mortality, may understate the true benefit of reducing pollution, as air pollution also affects scholastic achievement and human capital formation.

\section{KEY FINDINGS}

\section{Pros}

$\oplus$ Better air quality conditions are linked with improved test scores and school attendance.

- School absences induced by air pollution may cause parents to miss work days and incur sizable costs to schools located in states where funding is dependent on attendance.

- Better air quality can improve matching between students and their subsequent occupations by reducing the distortionary effect of air pollution on the reliability of exams.

๑ Stronger air quality regulation in economically deprived areas can improve social mobility, as the parts of cities that are most exposed to pollution tend to be the poorest.

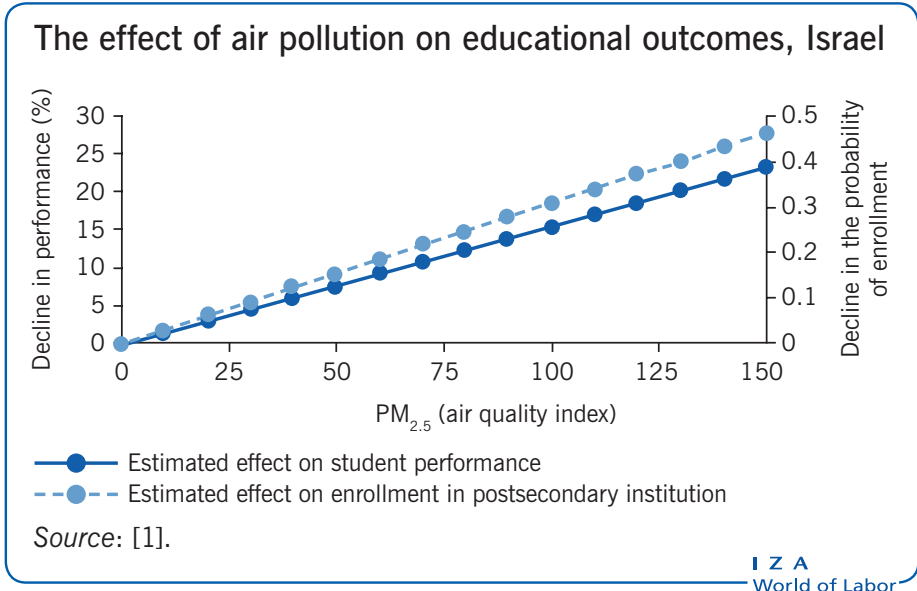

\section{Cons}

- Evidence on the expected monetized educational benefits of improving air quality is very limited.

- The precise underlying channels linking pollution with scholastic achievement are still not fully clear, which limits the ability to construct efficient policy responses.

- Stricter air pollution regulations may impose additional production costs, reduce competitiveness among affected firms, and reduce workers' earnings.

\section{AUTHOR'S MAIN MESSAGE}

Recent empirical evidence implies that air pollution imposes higher costs on society, because it adversely affects scholastic achievement and human capital formation, in addition to human health. Furthermore, improving air quality may also improve social mobility, as evidence suggests that air pollution affects educational outcomes unevenly across the income distribution. Hence, policymakers should acknowledge these additional effects when formulating new environmental regulations and should revisit existing policies, which are based on the impacts of pollution on human health alone. 


\section{MOTIVATION}

Recent data from the World Health Organization (WHO) show that ambient air pollution has risen in hundreds of already blighted urban areas across the globe. The well-known adverse human health consequences from exposure to elevated levels of pollution have led the WHO to describe the situation as a "public health emergency." However, air pollution may also affect aspects of human life beyond health, such as learning outcomes. This is of paramount importance, as a sound educational background is essential to many occupations. Therefore, it is vital to take these potential additional costs into account when formulating contemporary environmental policies.

The effect of air pollution may also have uneven impacts across the income distribution. This is due to various factors; for example, low-income individuals are more likely to live in highly polluted areas because of financial constraints or a lack of environmental awareness. Moreover, protective behaviors (such as the usage of preventive medications) to avoid or minimize the adverse effects of exposure to pollution are more prevalent among families from high social economics status (SES). Therefore, improving air quality in highly polluted and economically deprived areas may also improve social mobility, as air pollution might affect scholastic achievement and human capital formation unevenly.

\section{DISCUSSION OF PROS AND CONS}

\section{How air pollution may affect education}

There are several potential mechanisms that can link air pollution to educational outcomes. First, scholastic achievement may be affected directly by deterioration in oxygen quality induced by air pollution, as the brain consumes a large fraction of the oxygen needed by the body. Studies also suggest that exposure to air pollution affects brain development during childhood and fetal development. However, as stated by one group of researchers, "the possible neurodegenerative effect of air pollution remains largely unexplored" [2]. Another possible channel is via contemporaneous mild health conditions. More specifically, air pollution exposure may cause irritation of the eyes, nose, and throat, as well as asthma attacks, headaches, dizziness, and fatigue, which can all clearly affect student achievement. Finally, pollution may increase school absences, either due to pollution-related illness or parental decisions to keep children at home to avoid pollution exposure. This, in turn, may negatively affect educational achievement due to a reduction in formal contact time at school. It is important to note that the above mechanisms may also affect human capital formation (broadly defined as the skills, knowledge, and experience accumulated by an individual) and even labor market outcomes, since exams are often used as a primary method to assign students to further education and occupations.

\section{Methods to study air pollution's impact and limitations}

Epidemiological studies have found significant correlations between pollution exposure and cognitive performance. However, these studies mainly use cross-sectional data and, as such, do not necessarily reveal a causal link due to the potential presence of confounding factors. More specifically, there are several empirical challenges in estimating the causal 
effect of air pollution on educational outcomes. For example, air pollution is often correlated with other factors (such as wealth), which are also likely to be correlated with school or student characteristics, generating a potential omitted variable bias problem. Therefore, if wealthier families are sorting themselves into residential locations with lower levels of pollution, a naive ordinary least squares (OLS) analysis may overestimate the true effect of air pollution due to the positive association between wealth and education.

To address this and other related statistical concerns, applied econometricians tend to use quasi-experimental designs, which mainly focus on within-school or student variation. This lends further support to the causal interpretation of their analysis, as it relies on comparing students within the same school, or even comparing the same student over time. More generally, this type of analysis controls for all observed and unobserved potential confounders that remain constant over time. Another form of quasi-experimental design that applied researchers often use is a policy change that induces variation in pollution exposure only for a subsample of the population. For example, China's Huai River policy, which provided free winter heating via the provision of coal for boilers in cities north of the Huai River but did not provide it to cities south of the river, led to increased levels of air pollution in the north, as combustion of coal in boilers is associated with the release of air pollutants [3]. These exogenous events enable the researcher to construct control and treatment groups that are statistically identical apart from their pollution exposure. This form of study design can also be seen as a social analogue to a randomized experiment, which would clearly be unethical in this context.

Nevertheless, quasi-experimental designs also have their limitations. First, the results do not explicitly explain the underlying mechanism. Second, there could still be timevariant omitted variables that some of these models fail to capture. Third, the challenge of assigning pollution exposure to individuals may affect the reliability of the estimates. More specifically, the dilemma here is whether to assign pollution exposure to an individual's home location, school location, or some combination of the two. While this issue is less profound in younger populations (since they tend to go to schools in close proximity to their homes), it is still likely to yield some degree of measurement error due to the significant spatial variation in pollution, even within finely defined areas.

\section{Empirical evidence: The effect of parental and early life exposure}

Parental exposure to ambient air pollution during fetal development has been linked to various health outcomes, such as lower birth weights, increased prematurity, and congenital anomalies [4]. Several studies investigate additional outcomes beyond health. One of these examines whether in utero pollution exposure also affects long-term educational outcomes [5]. This study evaluates the impact of parental total suspended particulate (TSP) exposure during the year of birth on high school exams in Texas. To overcome the standard statistical concerns, the author uses county-level variation in the timing and severity of the industrial recession of the early 1980s as an exogenous shock to ambient TSPs. More specifically, the author exploits the fact that the industrial recession in Texas from 1981 to 1983 led to dramatic differential declines in manufacturing production and associated ambient TSP levels across geographical regions. The results suggest that parental exposure to TSP during the year of birth has a statistically significant effect on high school exit exams. 


\section{Understanding pollution terminology}

Air pollution can be defined as the presence of harmful substances in the air that interfere with human health and ecosystems. There are a handful of commonly found pollutants that are regulated by agencies around the world (such as the US Environmental Protection Agency and the EU), mainly due to their significant adverse effects on human well-being. One such criteria air pollutant is particulate matter (PM), which is a mixture of solid particles and liquid droplets suspended in the air that can consist of various components, including acids, metals, dust particles, and organic chemicals.

Particle pollution is classified into three main categories based on their size, namely: total suspended particulate (TSP), inhalable coarse particles $\left(\mathrm{PM}_{10}\right)$, and fine particles $\left(\mathrm{PM}_{2.5}\right)$. $\mathrm{PM}_{2.5}$ corresponds to $\mathrm{PM}$ that is 2.5 micrometers or smaller in diameter, which is at least 30 times less than the width of an average human hair. This is extremely important as the size of the particle is associated with its ability to cause health problems. In fact, exposure to PM can lead to severe respiratory and cardiovascular conditions such as heart attacks, decreased lung function, and even premature deaths, but also to milder conditions such as irritation of the eyes and throat, headaches, and dizziness.

There are many outdoor sources of particles including power plants, factories, mining, forest fires, and automobiles. However, it is important to note that indoor concentrations of particles are not simply a byproduct of ambient pollution; they are also the result of emissions from indoor sources such as cooking stoves, cleaning products, and various building materials. Interestingly, studies show that indoor concentrations of particles can significantly surpass outdoor levels, especially in venues with inadequate ventilation.

Another study also examines the impact of fetal exposure to air pollution on scholastic achievement, but in a developing country context [6]. This is of particular importance, as air pollution tends to be higher in developing countries (see Figure 1). The authors analyze this link by relying on sibling comparison in conjunction with controls for air quality alerts, which allow them to address concerns regarding confounding factors such as locational sorting and short-term time-varying protective behavior. They find a

Figure 1. Estimates of global fine particulate matter $\left(\mathrm{PM}_{2.5}\right)$ concentrations

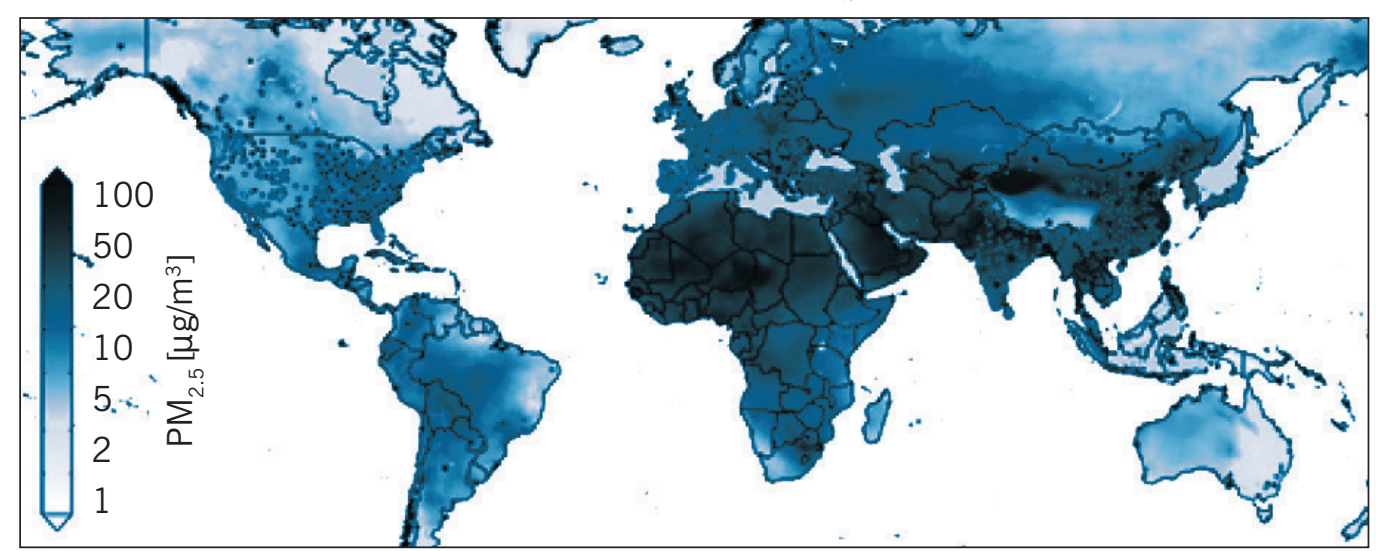

Source: Atmospheric Composition Analysis Group. Online at: http://fizz.phys.dal.ca/ atmos/martin/?page_id=140. Estimates based on information detailed in Van Donkelaar, A., R. V. Martin, M. Brauer, N. C. Hsu, R. A. Kahn, R. C. Levy, A. Lyapustin, A. M. Sayer, and D. M. Winker. "Global estimates of fine particulate matter using a combined geophysicalstatistical method with information from satellites, models, and monitors." Environmental Science \& Technology 50:7 (2016): 3762-3772. 
strong negative association between fetal exposure to carbon monoxide (CO) and lower mathematics and language skills among fourth grade students in Santiago, Chile.

A third study examines a similar link by looking at the relationship between fetal exposure to radically elevated levels of ambient pollution and long-term educational outcomes [7]. This study uses the severe air pollution episode that affected London for five days in December 1952 as an exogenous shock to in utero exposure to ambient pollution. The analysis, which focuses on individuals observed in 2001 and 2011, suggests that the children of the mothers who were affected by the severe air pollution in 1952 were 3\% less likely to have a high school qualification (A-level) compared to their counterparts who were not affected by the smog.

Finally, one researcher exploits the introduction of regulations to phase out the use of leaded gasoline in Sweden to investigate the effect of reduced early childhood lead exposure on educational and labor market outcomes [8]. The analysis is mainly based on differential changes in exposure to lead across birth cohorts within the same municipalities, which was induced by the regulations. Overall, the results show that reduced lead exposure early in life significantly improves scholastic performance, cognitive ability, and labor market outcomes in adulthood. Furthermore, the effect is stronger among low-SES children, suggesting that intergenerational correlations in economic outcomes may be reduced by environmental policies.

\section{The effect of short-term exposure}

Researchers have documented that short-term exposure to ambient air pollution decreases circulatory performance and leads to increased hospitalization rates. Recent studies have also documented a causal link between ambient pollution and reduced worker productivity. These observed physiological effects in conjunction with theoretical pathways from the medical literature suggest that short-term exposure to ambient air pollution may also affect educational outcomes.

One group of researchers examines this potential link among elementary school children in California, US [9]. More specifically, they study how changes in ambient air pollution concentrations near schools in Los Angeles affect the performance of second through sixth grade students on standardized tests. To account for potential confounders, they rely on within-grade-school variation (i.e. different cohorts within the same school) and a number of time-changing control variables. They find that a reduction in particulate matter (especially coarse particulate $\left(\mathrm{PM}_{10}\right)$ ) significantly increases mathematics and reading test scores.

Another study also examines this relationship by estimating the effect of fine particulate matter $\left(\mathrm{PM}_{2.5}\right)$ on scholastic performance of students in standardized exams in Israel [1], which are taken on multiple days in the same location after each grade (see illustration on page 1). The authors are able to exploit these in order to explore the impact of pollution by looking at the varying performance of individual students across the exam period. The results demonstrate that taking an exam on a polluted day was associated with a $3.2 \%$ decline in a student's test scores, with boys and students from low socio-economic backgrounds being most affected. Importantly, they find that this effect is evident at pollution levels below current environmental standards. The study also suggests that exacerbation of respiratory health problems, such as asthma, could be the mechanism for the negative relationship between air pollution and test outcomes. 
The authors then examine the long-term consequences of short-term exposures to ambient pollution. More specifically, they study how these short-term exposures affect human capital formation (which is measured by postsecondary educational attainment) and earnings of the same test-taking students during adulthood. The results show that those students who sat exams on high pollution days had a lower chance of obtaining a place at university compared with their unaffected counterparts, and completed fewer years of postsecondary education. The study also finds that short-term exposure to as little as an additional 10 units of $\mathrm{PM}_{2.5}$ (Air Quality Index) during the matriculation exams is associated with a $2.1 \%$ decline in monthly earnings in adulthood. Figure 2 demonstrates the negative relationship between residual $\mathrm{PM}_{2.5}$ exposure during the matriculation exams and residual test scores across quantiles of pollution exposure.

Figure 2. Impact of pollution exposure during high school exit exams on wages later in life, Israel

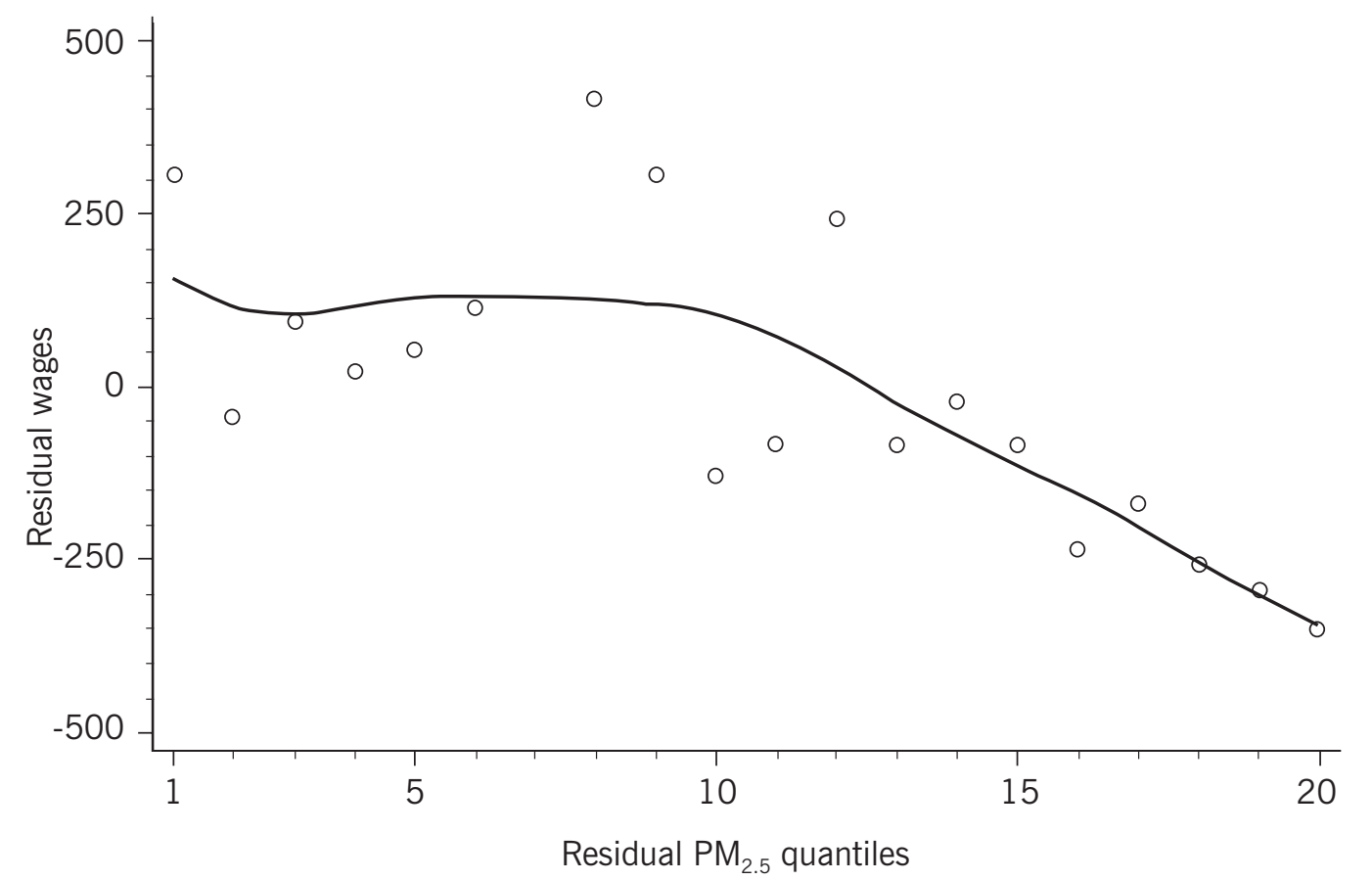

Note: The axes show quantiles of residual $\mathrm{PM}_{2.5}$ and residuals of wages in order to capture within-school variation (students who go to the same school but are exposed to different levels of pollution).

Source: Ebenstein, A., V. Lavy, and S. Roth. "The long-run economic consequences of high-stakes examinations: Evidence from transitory variation in pollution." American Economic Journal: Applied Economics 8:4 (2016): 36-65 [1].

Overall, the study highlights that short-term exposure to $\mathrm{PM}_{2.5}$ has a statistically and economically significant effect on student performance, and that in the context of highstakes exams this may also have significant long-term economic consequences. This longterm analysis is of great importance, as it reveals a monetary estimation for some of the costs associated with reduced student performance induced by air pollution, and supports the claim that pollution affects human capital formation as well as short-term scholastic achievement. Furthermore, the study suggests that by temporarily reducing student performance, elevated levels of pollution may lead to inefficient allocation of 
workers across occupations, as high-stakes testing is widely used to rank students and consequently assign them to further training and occupations. The incorrect ranking of students may also reduce labor productivity due to the poor assignment of workers to different occupations, as students with lower human capital are assigned a higher rank than their more qualified peers.

\section{Longer-term exposure}

There are very few studies that examine the link between longer time scales of students' pollution exposure and their educational outcomes. One possible pathway that can lead to such an association is that air pollution-related health conditions (such as aggravated asthma) may cause students to miss school days. One US study that examines this potential link uses data on steel mill opening and closing in the Utah Valley [10]. The authors estimate that an increase of 100 micrograms of $\mathrm{PM}_{10}$ increases school absence by $40 \%$.

Another group of researchers also study this link by using administrative data for school children in Texas [11]. They address many of the identification concerns by relying on variation at the school-year-period level in conjunction with a rich set of time varying controls. The results suggest that elevated levels of CO significantly increase absences among elementary and middle school children. As with the study on Israeli students, the authors find that this effect is present at levels well below current US Environmental Protection Agency standards.

The strong link between pollution and school absence suggests that improving air quality may improve educational outcomes via increased contact time at school. However, the full economic benefits of reduced school absences are probably even larger. This is because school absence may cause parents to miss work days and incur considerable costs to schools located in states where funding is dependent on attendance.

There are reasons to believe that longer-term exposure may also affect schooling via other channels. For example, repeated exposure to air pollution may affect cognition and therefore scholastic achievement. The study on Israeli students examines this possibility when looking at the relationship between $\mathrm{PM}_{2.5}$ exposure during exams at the tenth and eleventh grades and average test scores at the conclusion of the twelfth grade [1]. The authors find no evidence that such a link exists, but it is important to emphasize that their analysis relies on exposures during previous exams and not during the entire academic year.

\section{Indoor air pollution}

While most of the studies in the literature focus on ambient air pollution, two recent papers aim to examine the effect of indoor air pollution on educational outcomes. This is of particular importance because indoor air pollution is not simply a byproduct of ambient pollution. While some outdoor pollutants (mainly fine particles) can travel through small passageways, and therefore affect pollution concentrations inside classrooms, there are also many indoor sources of emissions, such as cleaning products, various building materials, and even personal care products. Hence, it is unclear whether 
previous studies that looked at the contemporaneous effect of air pollution captured the effect of pollution on the way to the exam, during the exam, or some combination of the two. It is therefore important to distinguish among these potential channels as the adequate policy response may vary considerably.

A recent study examines this potential link between indoor air quality (IAQ) and academic outcomes by looking at elementary school children in the US [12]. The study exploits a large renovation project that was designed to improve school IAQ at almost every elementary school within one school district in Texas. The exogenous variation comes from the different timing of renovation projects across schools. The study shows that IAQ renovations have a significant positive effect on standardized tests scores and no effect on student attendance. The study also indicates that IAQ-related renovations may be a cost-effective way to improve test scores. However, one should note that this study does not use actual readings of IAQ, instead using renovations as a proxy. It is therefore hard to conclude whether the improved test scores are due to an improvement in air quality, improved material and aesthetic conditions, or both.

Another recent study manages to shed light directly on the relationship between actual indoor air pollution concentrations and scholastic achievement [13]. More specifically, it uses a unique data set that combines readings of indoor air pollution (specifically, $\mathrm{PM}_{10}$ ) with administrative data on 2,458 students taking 11,522 exams in a leading public research university in England. The study, which relies on within-student variation, finds that increased exposure to $\mathrm{PM}_{10}$ significantly decreases test scores, especially among male, high ability, and science, technology, engineering, and mathematics (STEM) students. The results are robust to various linear and non-linear specifications and further indicate that the effect is present mainly in pollution levels above the WHO standards.

\section{LIMITATIONS AND GAPS}

The evidence on the causal link between air pollution and educational outcomes is still limited to a small number of studies, which are almost entirely conducted in developed countries. It appears that there is only one credible source of evidence for such a causal link in developing countries, where air pollution tends to be significantly higher. This, in conjunction with the substantial variation in educational conditions across countries, means there is considerable scope to explore this link in the developing world.

Furthermore, the pillar of research that examines the relationship between short-term exposure to air pollution and student performance has failed to provide convincing evidence for the underlying mechanism. Indeed, some suggestive evidence does exist, and proposes that the results may be due to exacerbation of respiratory health problems. However, the precise channels remain unclear. This is of particular importance, as different mechanisms may require different policy responses.

Finally, the literature reveals a strong and significant relationship between air pollution and education, which highlights the potential need for tighter environmental regulations. However, there is very limited and incomplete evidence on the associated costs of air pollution on education, which is crucial for policy making. To estimate these costs, researchers need access to large and long-term individual level data, with very detailed information on educational outcomes, demographics, and earnings. Nevertheless, it is 
important to emphasize that stricter environmental regulations could introduce their own costs. More specifically, tighter regulations may increase production costs via investment in abatement technology, reduce competitiveness of affected firms (especially those exposed to trade), and negatively affect employment and earnings.

\section{SUMMARY AND POLICY ADVICE}

The empirical evidence on the relationship between air pollution and educational outcomes has been growing in recent years. This, in conjunction with the increased use of credible quasi-experimental methods, suggests that the link is causal, and highlights that the traditional narrow focus on health outcomes (such as morbidity and mortality) may understate the true cost of pollution. Furthermore, the empirical evidence suggests that these effects exist at pollution levels well below current environmental standards. Therefore, there is a need for tighter environmental policies compared to current regulations, which are based solely on acute human health, given that pollution also affects scholastic achievement and human capital formation.

To determine the optimal policy intervention, regulators must understand the net welfare gains from improving air quality. However, while the expected health benefits and costs of environmental regulation have been studied extensively, credible estimates regarding the monetized educational benefits associated with improving air quality are extremely scarce. Policymakers should therefore support scientific research on the issue and base their decisions on a fully-fledged cost-benefit analysis, which takes into account these additional factors.

Finally, air pollution is not randomly assigned across areas, and those who suffer the most tend to be residents of the poorest parts of cities. This is mainly because of their proximity to factories and other disamenities, which makes them more exposed to air pollution. Therefore, improving air quality may have a particularly great effect on underprivileged students, potentially enhancing their ability to achieve positive socio-economic mobility.

\section{Acknowledgments}

The author thanks two anonymous referees and the IZA World of Labor editors for many helpful suggestions on earlier drafts. Previous work of the author contains a larger number of background references for the material presented here and has been used intensively in parts of this article [1], [13]. The author would also like to thank Ghazala Azmat.

\section{Competing interests}

The IZA World of Labor project is committed to the IZA Guiding Principles of Research Integrity. The author declares to have observed these principles.

(c) Sefi Roth 


\section{REFERENCES}

\section{Further reading}

Pope III, C. A., and D. W. Dockery. "Health effects of fine particulate air pollution: Lines that connect." Journal of the Air and Waste Management Association 56:6 (2006): 709-742.

Isen, A., M. Rossin-Slater, and W. R. Walker. "Every breath you take-Every dollar you'll make: The longterm consequences of the Clean Air Act of 1970." Journal of Political Economy 125:3 (2017): 848-902.

\section{Key references}

[1] Ebenstein, A., V. Lavy, and S. Roth. "The long-run economic consequences of high-stakes examinations: Evidence from transitory variation in pollution." American Economic Journal: Applied Economics 8:4 (2016): 36-65.

[2] Suglia, S. F., A. Gryparis, R. O. Wright, J. Schwartz, and R. J. Wright. "Association of black carbon with cognition among children in a prospective birth cohort study." American Journal of Epidemiology 167:3 (2008): 280-286.

[3] Chen, Y., A. Ebenstein, M. Greenstone, and H. Li. "Evidence on the impact of sustained exposure to air pollution on life expectancy from China's Huai River policy." Proceedings of the National Academy of Sciences 110:32 (2013): 12936-12941.

[4] Currie, J., and R. Walker. "Traffic congestion and infant health: Evidence from E-ZPass." American Economic Journal: Applied Economics 3:1 (2011): 65-90.

[5] Sanders, N. J. "What doesn't kill you makes you weaker: Prenatal pollution exposure and educational outcomes.” Journal of Human Resources 47:3 (2012): 826-850.

[6] Bharadwaj, P., M. Gibson, M., J. Graff Zivin, and C. A. Neilson. "Gray matters: Fetal pollution exposure and human capital formation." Journal of the Association of Environmental and Resource Economists 4:2 (2017): 505-542.

[7] Ball, A. Air Pollution, Foetal Mortality, and Long-Term Health: Evidence from the Great London Smog. MBRA Paper No. 63229, November 2014.

[8] Nilsson, J. P. The Long-Term Effects of Early Childhood Lead Exposure: Evidence from the PhaseOut of Leaded Gasoline. IFAU Working Paper, November 2009.

[9] Ham, J. C., J. S. Zweig, and E. Avol. Pollution, Test Scores and the Distribution of Academic Achievement: Evidence from California Schools 2002-2008. IZA Conference Paper, October 2011.

[10] Ransom, M. R., and C. A. Pope. "Elementary school absences and $\mathrm{PM}_{10}$ pollution in Utah Valley." Environmental Research 58:1 (1992): 204-219.

[11] Currie, J., E. A. Hanushek, E. M. Kahn, M. Neidell, and S. G. Rivkin. "Does pollution increase school absences?" The Review of Economics and Statistics 91:4 (2009): 682-694.

[12] Stafford, T. M. "Indoor air quality and academic performance." Journal of Environmental Economics and Management 70 (2015): 34-50.

[13] Roth, S. The Contemporaneous Effect of Indoor Air Pollution on Cognitive Performance: Evidence from the UK. LSE Miemo, 2016.

\section{Online extras}

The full reference list for this article is available from:

http://wol.iza.org/articles/air-pollution-educational-achievements-and-human-capital-formation

View the evidence map for this article:

http://wol.iza.org/articles/air-pollution-educational-achievements-and-human-capital-formation/ map 